\title{
Immediate heart-rate response to standing: simple test for autonomic neuropathy in diabetes
}

\author{
D J EWING, I W CAMPBELL, A MURRAY, J M M NEILSON, B F CLARKE
}

British Medical fournal, 1978, 1, 145-147

\section{Summary and conclusions}

The immediate heart-rate response to standing was measured in 22 normal controls and 25 patients with diabetes, 15 of whom had autonomic neuropathy. The response in the controls and patients without autonomic neuropathy was characteristic and consistent, with tachycardia maximal at around the 15th beat and relative bradycardia maximal at around the 30th beat. The diabetics with autonomic neuropathy, however, showed a flat response. In three controls the response was abolished with intravenous atropine but not with propranolol, showing that it is mediated through the vagus.

A simplified test using routine ECGs and measuring the $R-R$ interval at beats 15 and 30 with a ruler is easily performed as an outpatient procedure and may be used as a measure of autonomic function in diabetes.

\section{Introduction}

The integrity of the autonomic nervous system may be assessed with several simple tests based on cardiovascular reflexes. ${ }^{1}$ These tests, however, have disadvantages. Responses to the Valsalva manoeuvre ${ }^{2}$ and the sustained-handgrip test ${ }^{3}$ depend on effort, and the postural fall in blood pressure may be unreliable in patients with fluid retention, who may have extensive autonomic damage without postural hypotension. ${ }^{4}$ Furthermore, in diabetes the blood-pressure response to posture varies throughout the day and may be related to the timing of insulin injections. ${ }^{5}$ The assessment of variation in heart rate (or $R-R$ interval) both at rest and during deep breathing is simple and objective but requires special equipment. ${ }^{6}$ Most other tests are either invasive or cause discomfort.

Changing from lying to standing produces an integrated reflex response of the cardiovascular system, which includes alterations in heart rate and blood pressure. ${ }^{1}$ We describe a computer analysis of the immediate heart-rate response to standing in normal and diabetic subjects and its application using an electrocardiogram (ECG) as a simple, new test of autonomic function that can be used as an outpatient procedure.

\section{Subjects and methods}

We studied 22 normal men and 25 patients with diabetes. The normal men comprised 12 "young controls"-namely, laboratory

\footnotetext{
University Departments of Medicine and Medical Physics, and the Diabetic and Dietetic Department, Royal Infirmary, Edinburgh EH3 9YW

D J EWING, MD, MRCP, lecturer in medicine

I W CAMPBELL, MB, MRCP, senior registrar

A MURRAY, PHD, senior physicist (present address: Department of Cardiology, Newcastle General Hospital)

J M M NEILSON, PHD, senior lecturer in medical physics

B F CLARKE, MB, FRCP, consultant physician and senior lecturer in medicine
}

personnel aged 21-45 (mean 28) years-and 10 "older controls"namely, men aged 48-67 (mean 56) years who had attended a heartdisease-prevention trial and had no evidence of cardiovascular or respiratory abnormalities. Of the diabetics, 10 (three men and seven women) aged 21-51 (mean 34) years were insulin-dependent and had been diagnosed 7-16 (mean 11) years previously. None had evidence of autonomic neuropathy or postural hypotension, and all gave a normal response to the Valsalva manoeuvre. The remaining 15 diabetics (13 men and two women), aged 28-71 (mean 49) years, had been diagnosed 4-36 (mean 20) years previously. All had evidence of autonomic neuropathy (reduced $R-R$ interval variation and abnormal responses to the Valsalva manoeuvre and sustained-handgrip test) and postural hypotension. Thirteen were insulin-dependent and two were receiving chlorpropamide.

\section{COMPUTER ECG ANALYSIS}

Each subject lay quietly for three minutes, then stood up (within five seconds) and remained motionless for two minutes. A continuous ECG was recorded on magnetic tape for later analysis, and a signal marker was put into a second channel of the tape-recorder to identify the time at which the subjects had begun to stand. The tape was replayed at 60 times real speed, and a graphical and numerical playout of the individual $R-R$ interval measurements obtained, as described. ${ }^{7}$ For analysis of the grouped data the measurements at every fifth beat after standing were used.

\section{ROUTINE ECG ANALYSIS}

To see whether the analysis could be simplified, a direct-writing ECG was recorded simultaneously with the tape-recording for 10 of the controls and 11 of the diabetics (seven with autonomic neuropathy). A marker was used to identify the time of standing, although a characteristic muscle tremor usually appeared on the ECG as the subject stood up. The ECG was recorded from about 15 beats before to 40 beats after standing. The R-R intervals at beats 15 and 30 after standing were measured with a ruler.

\section{PHARMACOLOGICAL STUDIES}

Three of the controls (all medical staff) had their lying-to-standing heart rates recorded before and after the administration of intravenous atropine $3 \mathrm{mg}$ and propranolol $10 \mathrm{mg}$.

\section{Results}

\section{COMPUTER ECG ANALYSIS}

All but one of the controls showed a characteristic and consistent response to standing, with tachycardia (measured as a shortened R-R interval) maximal at about 10 seconds-that is, between beats 10 and 20 -and relative bradycardia (lengthened $R-R$ interval) maximal at about 20 seconds-that is, between beats 25 and 35 (fig 1). The diabetics with no evidence of autonomic neuropathy had responses similar to those of the controls. In contrast, the diabetics with autonomic neuropathy showed a flat response, with only a small increase in heart rate and no relative bradycardia (fig 2). Fig 3 gives the mean results for all four groups of subjects.

In the tracings obtained from the controls the maximal tachycardia occurred at around the 15 th beat, while the subsequent relative bradycardia was maximal at around the 30th beat. A simple ratio, the " $30: 15$ ratio" (length of R-R interval at beat 30 divided by length of $R-R$ interval at beat 15 ), was used to express the response numerically; 


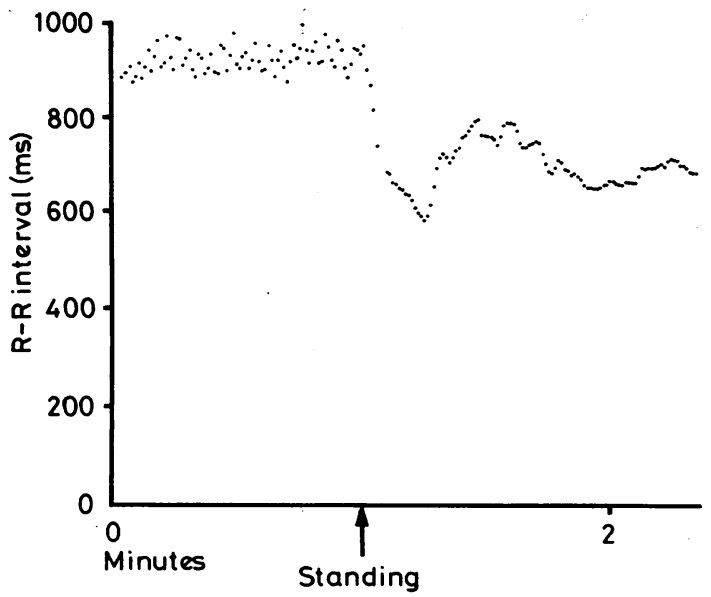

FIG 1 -Response of $R-R$ interval to standing in normal 31-year-old man.

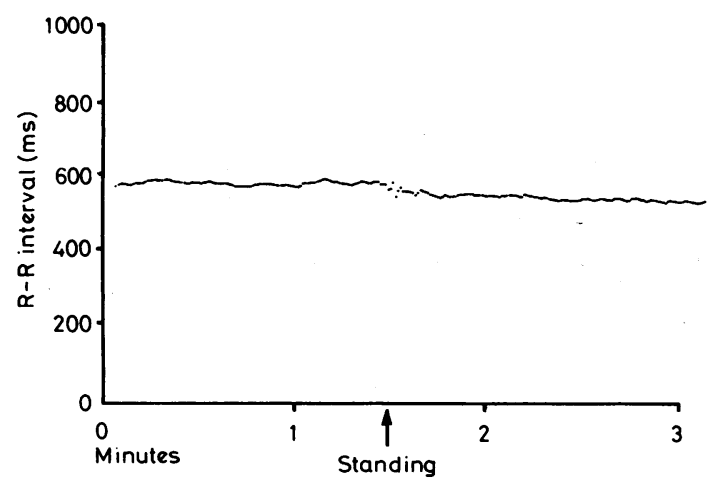

FIG 2-Response of $\mathbf{R}-\mathbf{R}$ interval to standing in 30-year-old man with diabetic autonomic neuropathy.

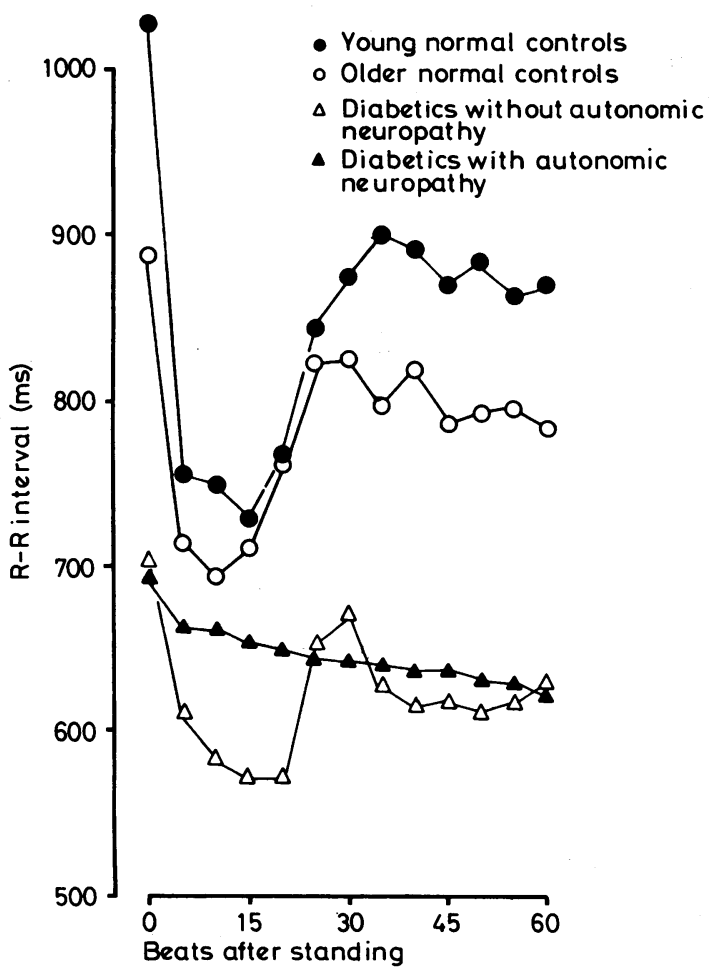

FTG 3-Mean R-R intervals in the four groups studied, measured every fifth beat for 60 beats. the individual results are given in fig 4. All but one of the controls and all of the diabetics without autonomic neuropathy had ratios of 1.03 or greater. All 15 diabetics with autonomic neuropathy had ratios less than 1.03 , and 12 had ratios of 1.00 or less. The $30: 15$ ratio in the controls was not related to age or resting heart rate.

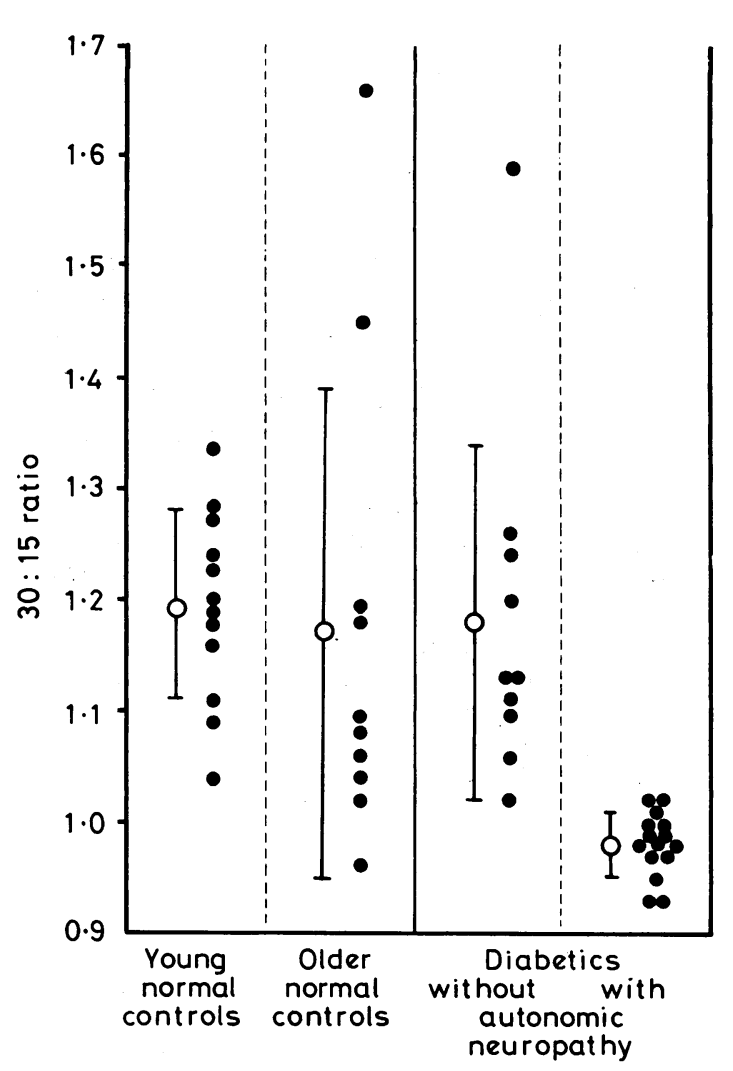

FIG 4-Individual 30:15 ratios in the four groups studied. Open circle and bars in each group represent mean $\pm 1 \mathrm{SD}$.

\section{ROUTINE ECG ANALYSIS}

The 21 subjects whose responses were recorded simultaneously on magnetic tape and by routine electrocardingraphy had their 30:15 ratios calculated from each record. The results showed a close correlation $(r=0 \cdot 89)$. Five young controls then performed the test five times on different days, and the 30:15 ratio was found to be reproducible (coefficient of variation $5.3 \%$ ). Six diabetics with autonomic neuropathy repeated the test after three months, and the results were unchanged.

\section{PHARMACOLOGICAL STUDIES}

The characteristic heart-rate response in the three controls was abolished with intravenous atropine and with combined intravenous atropine and propranolol, but not with intravenous propranolol alone, showing that the response is mediated through the vagus.

\section{Discussion}

We found a striking difference in the immediate heart-rate response to standing between normal subjects and diabetics with autonomic neuropathy. All the controls and diabetics without autonomic neuropathy had a characteristic rapid increase in heart rate over the first few beats, followed by a plateau for a further 5-10 beats, and then a relative bradycardia which was maximal at about the 30 th beat. In contrast the diabetics with autonomic neuropathy showed no such response, their heart 
rates increasing only slightly over the same 30 -beat period. These diabetics had other evidence of vagal damage, with abnormal responses to the Valsalva manoeuvre and reduced $R-R$ interval variation. The results of the drug studies on the three controls confirm that the reflex is mediated through the vagus.

In $1895 \mathrm{Hill}^{8}$ suggested that changes in posture might provide a "most delicate test of the condition of the vasomotor mechanism," yet surprisingly little attention has been paid to the normal heart-rate response to standing. It is well recognised that there is a transient fall in blood pressure on standing, with stimulation of the carotid baroreceptors and consequent reflex tachycardia and peripheral vasoconstriction. ${ }^{1}$ Although it has long been known that in normal people the heart rate increases on standing, ${ }^{8}$ the immediate heart-rate response has only recently been briefly documented. ${ }^{9}$ So far as we are aware, the characteristic pattern that we describe has not previously been analysed in detail.

Although our results were first obtained from an accurate $\mathrm{R}-\mathrm{R}$ interval analysis by computer, this study shows that heartrate changes may also be detected with routine electrocardiography. As loss of a normal response is due to vagal damage, this provides the basis for a simple test of autonomic function that has considerable advantages over those now in use. Measurement of the 30:15 ratio gives a simple numerical value that reflects the presence or absence of the relative bradycardia. When the ratio is 1.00 or less vagal damage is probably present, although a value of less than 1.00 does not necessarily indicate more severe damage, as it will occur when there is a slight increase in heart rate over the 30 -beat period. In the most severe cases, in which there is no change in heart rate on standing, the value will be exactly $1 \cdot 00$.

This test is simple to use and requires only a standard electrocardiograph and the ability of the patient to stand up. It is not effort-dependent and, so far as we know, cannot readily be "cheated." It correlates well with other recognised tests of cardiovascular reflex function in diabetes, is objective, requires no special patient co-operation, and is readily applicable as an outpatient procedure.

We thank Dr W G Macfie for allowing us to study subjects from a primary prevention trial of ischaemic heart disease, and $\operatorname{Dr} P$ J Watkins for his initial suggestion that we should look at this reflex response.

\section{References}

1 Johnson, R H, and Spalding, J M K, in Disorders of the Autonomic Nervous System, p 33. Oxford, Blackwell, 1974.

2 Ewing, D J, et al, Lancet, 1973, 2, 1374.

${ }^{3}$ Ewing, D J, et al, Clinical Science and Molecular Medicine, 1974, 46, 295.

Campbell, I W, Ewing, D J, and Clarke, B F, British Medical fournal, 1976, 1, 872.

5 Page, $M$ M, and Watkins, $P$ J, Diabetes, 1976, 25, 90.

6 Wheeler, T, and Watkins, P J, British Medical fournal, 1973, 4, 584.

${ }^{7}$ Murray, A, et al, British Heart fournal, 1975, 37, 882.

${ }^{8}$ Hill, J, fournal of Physiology, 1895, 18, 15.

${ }^{9}$ Page, M M, and Watkins, P J, Clinics in Endocrinology and Metabolism, $1977,6,377$.

(Accepted 28 October 1977)

\section{SHORT REPORTS}

\section{Functions of phagocytic cells in chronic mucocutaneous candidiasis}

Chronic mucocutaneous candidiasis (CMC) is a group of uncommon immunodeficiency disorders characterised by chronic infection of the skin, nails, and mucosal surfaces caused by Candida albicans. There are few reports about the functions of phagocytic cells in CMC. ${ }^{1-3}$ This led us to investigate phagocytosis and intracellular killing of candida by granulocytes and monocytes in CMC with a recentlydeveloped technique.

\section{Patients, methods, and results}

Leucocyte functions were tested in five patients (two men (cases 2 and 5) and three women $(1,3$, and 4)) with CMC. In four of these patients the candida infection was limited to the skin, nails, and mucous membranes; the fifth patient (case 1) also suffered from recurrent bacterial infections (affecting the respiratory tract) and had the most severe candidiasis. Case 4 also suffered from hypoadrenalism and hypoparathyroidism.

The method for measuring phagocytosis and intracellular killing by $C$ albicans has been reported. ${ }^{4}$ To prevent a decrease in the number of the yeast cells caused by clumping of the yeast during the test period $C$ albicans was precultured for at least five days at $30^{\circ} \mathrm{C}$. Phagocytosis was assessed from the decrease in the number of extracellular candida determined in a haemacytometer. The rate of intracellular killing was determined microbiologically from the decrease in the number of viable candida after lysis of the phagocytic cells.

In all patients phagocytosis of $C$ albicans by granulocytes was normal in the presence of both normal serum and the patient's serum. Phagocytosis by monocytes of cases $1,2,4$, and 5 was in a normal range (93.8-98.9\%), whereas the monocytes of case 3 showed slightly diminished phagocytosis $(90.0 \%)$. The intracellular killing by granulocytes in the presence of normal serum or patient's serum was normal in all patients.

In the presence of normal serum the intracellular killing by monocytes was normal in two patients (cases 1 and 2), whereas two patients (4 and 5) showed hyperactive killing ( $>70 \%$ at 60 minutes) (see figure). Case 3 showed a slightly diminished killing. When patient's monocytes were used together with patient's serum, however, both cases 1 and 2 showed diminished intracellular killing and the results for cases 3,4 , and 5 were not altered (see

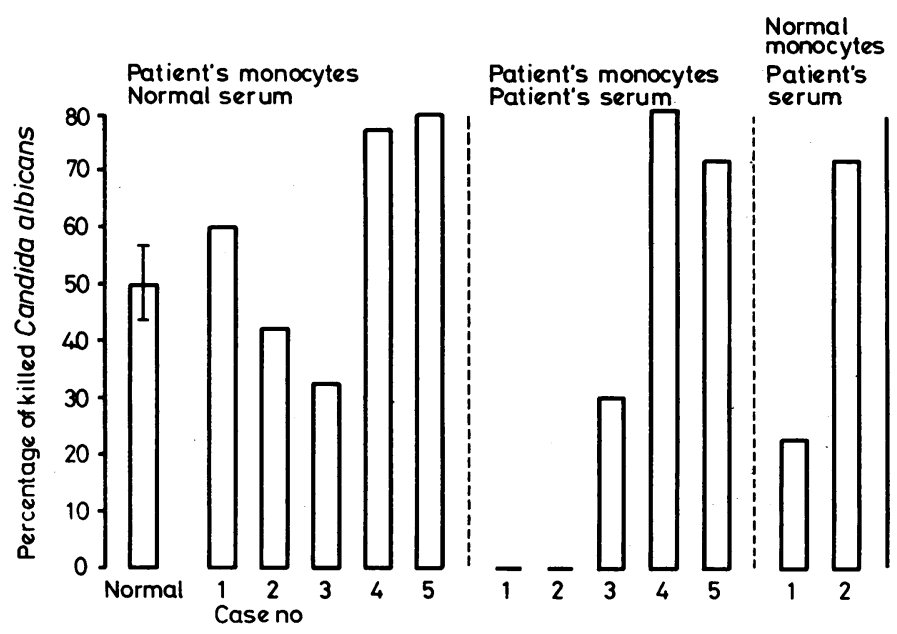

Intracellular killing of Candida albicans by human monocytes.

figure). When normal monocytes were used together with patient's serum, serum of case 2 showed increased intracellular killing, whereas with the serum of case 1 the intracellular killing was still diminished (see figure).

\section{Comment}

Concerning the functions of phagocytic cells in CMC Valdimarsson et $a^{1}$ reported normal intracellular killing by granulocytes in five patients, using the dye exclusion method. ${ }^{5}$ This method has the disadvantage of subjective microscopical judgment of candida cells being stained. Phagocytosis and intracellular killing of bacteria by leucocytes was normal in a child with recurrent pyogenic infections, CMC, and defective neutrophil chemotaxis. ${ }^{2}$

We found that phagocytosis and intracellular killing by granulocytes were normal in all patients, and that phagocytosis by monocytes 